\title{
The Efficacy of Ranikhet Disease Vaccines Produced by Livestock Research Institute of Bangladesh
}

\author{
Sreebas Chandra Sarkar, Sukumar Saha*, Md. Mansurul Amin and Md. Golzar Hossain
}

Department of Microbiology and Hygiene, Faculty of Veterinary Science, Bangladesh Agricultural University, Mymensingh-2202, Bangladesh.

*Corresponding author's e-mail: sukumar94@yahoo.com

[Received: 21 April 2012, Revised: 01 May 2012, Accepted: 30 June 2012]

\begin{abstract}
A B S T R A C T
The study was conducted to investigate the efficacy of Baby chick Ranikhet Disease Vaccine (BCRDV) and Ranikhet Disease Vaccine (RDV) produced by the Livestock Research Institute (LRI), Mohakhali, Dhaka. For this experiment, 100 day-old-chick was purchased from Phinex Hatchery Ltd., Gazipur. The chicks $(n=100)$ were divided into two groups. In group A $(n=50)$, vaccination was performed twice with BCRDV at 2 and 21 days of age through intraocular route (i/o) followed by once with RDV at 60-day of age through intramuscular $(\mathrm{i} / \mathrm{m})$ route. Group B $(\mathrm{n}=50)$ was kept as unvaccinated control. The immunogenicity of the vaccine was evaluated by measuring the serum HI antibody titers at 1-, 20-, 36-, and 76-day of age, while the vaccine efficacy was examined by a challenge infection experiment with a velogenic field isolate of NDV as well as passive protection test. It was observed that the maternal antibody titers of the unvaccinated control group B gradually declined from day 1 to day 76 of age. Conversely, after primary and secondary vaccination with BCRDV, the levels of serum HI titer slightly increased in vaccinated group A compared with those in control group B. Finally administration of RDV resulted in a sharp increase in $\mathrm{HI}$ titer, leading to protection from challenge infection with virulent field virus as well as passive protection test. These results clearly demonstrated that a prime-booster immunization with BCRDV and RDV, both produced by LRI, is effective to protect chicken against Newcastle disease (ND).
\end{abstract}

Keywords: Ranikhet disease, Vaccine, HI, Antibody, Velogenic

C 2012 Microbes and Health. All rights reserved

\section{Introduction}

Newcastle disease (ND) is a fatal viral disease of poultry due to its high contagiousness and rapid spreading nature among chicken and other domestic and semi-domestic species of birds and is endemic in Bangladesh. Strains of NDV are distinguished into five pathotypes (Beard and Hanson, 1984): viscerotropic velogenic viruses (vvND), neurotropic velogenic viruses (nvND), mesogenic viruses, lentogenic viruses, and asymptomatic enteric viruses causing gut infection but no apparent disease.

The clinical signs include respiratory, alimentary and nervous system characterized by signs of depression, diarrhea, prostration, edema of the head and wattles. In the vvND, clinical signs often begin with restlessness, increased respiration ending with death. In some circumstances, with extremely virulent viruses the disease may result in sudden death (Cheville et al., 1972b; Brown et al., 1999).

Supplemental materials are available at- http://journal.bsvmph.org/

To cite this article: Sarkar SC, S Saha, MM Amin and MG Hossain, 2012. The efficacy of ranikhet disease vaccines produced by Livestock Research Institute of Bangladesh. Microbes Health, 1(1): 9-13.
According to Chowdhury et al. (1982), Newcastle disease is responsible for at least $40-60 \%$ of total mortality of poultry in Bangladesh and is responsible for high economic losses every year. Vaccination is being practiced to protect ND in Bangladesh. To protect the chickens against ND mostly the lentogenic BCRDV (F-strain), mesogenic RDV (Mukteswar) and other commercial vaccines are used in Bangladesh. In spite of regular vaccination, the disease has been found to appear in every year in the form of epidemic which causes $40-60 \%$ of the total mortality rate of poultry population in Bangladesh (Chowdhury et al., 1981). As a result these industries are not being developed as expectations as well as backyard poultry rearer are losing their interest to involve in poultry rearing. Zhuo et al. (1998) found out that, the possible cause of outbreaks in immunized flock were interference by maternal antibody, poor husbandry, improper vaccination, failure of maintenance of cold chain system and antigenic variation among the vaccine strains and field strains at timing of vaccination. On the other hand vaccines that are commonly used should be stored at $4-8^{\circ} \mathrm{C}$ to maintain their efficacy. However in rural areas of Bangladesh it is not possible to maintain cold chain system appropriately and since there are varieties of mode and means of transport and variation occurs in the maintenance of cooling system at all stages of District, Thana, Union, Village and Farm 
level. Due to this problem vaccination failure is still a common problem. Quality of vaccine should be ensured to reduce the vaccine failure cases. Keeping such consideration in view, research program was undertaken to investigate the immunogenicity and protective activity of BCRDV and RDV produced by LRI, Dhaka, Bangladesh.

\section{Materials and Methods}

\section{Newcastle disease vaccine and virus}

BCRDV (lentogenic, F-strain) and RDV (mesogenic, Mukteswar strain) were collected from Livestock Research Institute (LRI), Mohkhali, Dhaka. Virus used for challenge was obtained from the Department of Microbiology and Hygiene, BAU, Mymensingh.

\section{Chicken eggs}

Fertile eggs of indigenous hens were purchased from Bangladesh Agricultural University poultry farm. The eggs were incubated at $37^{\circ} \mathrm{C}$ temperature maintaining relative humidity of $85 \%$ for 10 days and well-developed active embryos were used for the propagation of challenge virus.

\section{Experimental chicks}

A total of 100 day-old Hisex chicks were collected from the Phenix Hatchery Ltd., Gazipur. The chicks were then reared in the experimental poultry shed of the Department of Microbiology and Hygiene, BAU, Mymensingh.

\section{Propagation of virus in embryonated chicken eggs}

Virus suspension was collected from the repository of the virology laboratory of the Department of Microbiology and Hygiene and was thawed and then treated with antibiotic (Gentamycin $1.0 \mathrm{mg} / \mathrm{ml}$ ) for bacteriological sterility and prepared as an inoculum of which $0.1 \mathrm{ml}$ was inoculated into each of five 10-day-old embryonated chicken eggs through allantoic sac (AS) route. The eggs were then incubated at $37^{\circ} \mathrm{C}$ and observed twice daily for mortality of the embryo. The embryos that died within 24 hours of inoculation were discarded. The embryos that died during the desired period of observation were chilled at $4-8^{\circ} \mathrm{C}$ for 1-2 hours to reduce the contamination of allantoic fluid (AF) with blood and other extraneous material during harvesting. Each egg was swabbed with cotton soaked with $70 \%$ alcohol to disinfect and remove any dust from the shells. The egg shell above the air space was removed and $\mathrm{AF}$ was collected aseptically with sterile syringe and needle into sterile containers. The presence of NDV in AF was tested by the slide HA test and AF which manifested HA-positive (clumping) NDV were collected (Cottral, 1978). This was served as a stock virus suspension for NT and protection test.

\section{Serum from blood samples}

To obtain serum, blood was collected by disposable syringe and needle and placed in slanting position for an hour at room temperature. Then the plunger from the syringe was removed and the serum was collected in a test tube by pouring. This was then centrifuged at $1500 \mathrm{rpm}$ for 15 minutes to obtain clear serum and transferred to a small vial and labeled. Vials containing serum were stored at $-20^{\circ} \mathrm{C}$ temperature until used.

\section{Haemagglutination (HA) test}

Micro haemagglutination test was performed in a V bott- om micro-well plate to determine HA units (4HA/50 $\mu \mathrm{l})$. This was carried out by two-fold serial dilutions of the viral suspension in a micro-well plate and then tested to determine an end point. For this purpose, $50 \mu 1$ of PBS was dispensed into each well of the micro-well plate. Then $50 \mu 1$ of test sample (virus) were placed in first well of each row of column 1 and then two-fold dilution was made up to column $11.50 \mu 1$ of $1 \%$ red blood cells were added to each well including column 12 . The control wells contain only PBS and red blood cells. A cover was placed on the plate. The plate was allowed to stand for 45 minutes at room temperature. The results were read and recorded in each well. All the control wells were HA negative. In HA negative case, a sharp buttoning of red blood cells at the bottom of the V-bottom well and in HA positive case, a hazy film of red blood cells, no button or a very a small button of red blood cells at the bottom of the V-bottom wells were showed in micro well plate. The HA of the virus was determined as the highest dilution of the virus which agglutinates the RBC in the test. 4HA of the virus was calculated from the $1 \mathrm{HA}$.

\section{Haemagglutination inhibition (HI) test}

The HI test was performed to determine the HI titers of the sera samples collected from the chickens. The HI titers of sera samples of control chicken were determined to measure the maternal antibody and its persistence. The test was conducted by using constant 4 HA unit antigen and increasing serum dilution method ( $\beta$-procedure) following the procedure described in Anon (1971). The Haemagglutination titer of HA antigen was first determined by HA test. The antigen was then diluted in PBS to yield 4 HA units per $50 \mu 1$ of suspension. For performing the HI test, two folds serial dilution of the serum was prepared. Then $50 \mu 1$ of antigen suspension containing 4 HA units was added into each well except well number 12 of A, B, C, D, $\mathrm{E}, \mathrm{F}, \mathrm{G}$ and $\mathrm{H}$ as marked on the plate and mixed thoroughly. Well number $12 \mathrm{kept}$ as control. The serum antigen mixture was then incubated for 10 minutes at room temperature. Then $50 \mu 1$ of $1 \%$ chicken RBC suspension was added into each well. Then the mixture was again kept at room temperature for 45-60 minutes. A compact mass of sediment cells covering the bottom of the plate was considered as positive for HI. The serum end point was determined as the highest dilution of serum, which inhibited the agglutination of the RBC in the test. The HI titer of each serum was corresponded to reciprocal of highest original dilution of serum inhibiting agglutination of chicken RBC completely.

\section{Determination of $\mathbf{E L D}_{50}$ of the challenge virus}

The aim of the titration was to measure the concentration of Newcastle disease virus in a suspension. To determine the Embryo Lethal Dose $50\left(\mathrm{ELD}_{50}\right)$ and neutralizing titer of suspension of field isolate of NDV, a series of two-fold dilutions were carried out following Reed and Muench (1938). From each dilution $0.1 \mathrm{ml}$ was inoculated each of five 10-day-old embryonated chicken eggs through allantoic sac (AS) route. One drop of each dilution was put to NA plate/slant to test for bacterial contamination and incubated for 24 hours at $37^{\circ} \mathrm{C}$. For each test, five embryos were kept as control. The embryos were then incubated at $37^{\circ} \mathrm{C}$ for ten days and candled twice daily to check embryo mortality. Embryos that died within 24 hour of inoculation were discarded and the mortality of the embryos was recorded. The embryos that died or survived during the 
period of observation were chilled at $4-8^{0} \mathrm{C}$ for $1-2$ hours before collection of allantoic fluid (AF). AF from each embryo was subjected to slide HA test to detect presence of virus. The embryos revealing hemorrhagic lesion in the body and the AF showing positive $\mathrm{HA}$ reaction were considered infected and recorded for calculation. The $\mathrm{ELD}_{50}$ of virus strain were calculated from the data obtained as the method described by Reed and Muench (1938).

\section{Protection test}

This test was conducted at 76 days of age of both vaccinated (after vaccination of primary, secondary with BCRDV and $3^{\text {rd }}$ time with RDV at day 2, 21 and 60 respectively) and unvaccinated control birds. Ten birds were randomly selected from vaccinated group $\mathrm{A}$ and another 10 birds obtained from unvaccinated group B. Then all the $(10+10=20)$ birds were challenged with virulent field isolate of NDV @ $0.1 \mathrm{ml}$ of $2 \mathrm{ELD}_{50}$ per bird intranasally to observe the protective potential against the ND.

\section{Passive protection test}

Two hundred micro liter of pooled serum from vaccinated and control chicken was mixed with $2 \mathrm{ELD}_{50}$ dose of NDV separately (immune and nonimmune serum) incubated at $37^{\circ} \mathrm{C}$ for 30 minute. These suspensions were administered intranasally $\left(200 \mu 1\right.$ serum $\left.+100 \mu 12 E^{2} D_{50}\right)$ to two groups of 10 chickens of 10 weeks of age and mortality was monitored for subsequent 10 days.

\section{Analysis of data}

The statistical analysis was performed using student's ttest. Significance in survival rates of chicken were analyzed by Mantel-Cox log rank test. A $P$ value of $<0.05$ is being considered as statistically significant.

\section{Results}

\section{HI test}

To evaluate the efficacy of BCRDV and RDV, 100 dayold Hi-sex white chicks were divided into two groups of which Group A contain $(\mathrm{n}=50)$ vaccinated and Group B contain $(n=50)$ unvaccinated control. To evaluate immunogenicity, birds were vaccinated with BCRDV on day 2 and 21 through intraocular (i/o) route, one drop per bird and with RDV at day 60 through intramuscular (i/m) route, $1 \mathrm{ml}$ per bird. In order to determine serum $\mathrm{HI}$ titers in vaccinated group and any maternal antibody in control group, sera samples were obtained at 1, 20, 36 and 76 days of age of birds. In case of unvaccinated control group B HI titers (Mean \pm SD) were $70.40 \pm 035.054$, $17.60 \pm 8.76,9.60 \pm 3.57$, and $4.80 \pm 1.78$ at $1,20,36$ and 76 days of age respectively. These HI titers $(\mathrm{Mean} \pm \mathrm{SD})$ were converted into $\log _{2} \mathrm{HI}$ titers $(\mathrm{Mean} \pm \mathrm{SD})$ and were $6.00 \pm 0.7071, \quad 4.00 \pm 0.7071, \quad 3.20 \pm 0.4472$, and $2.20 \pm$ 0.4472 , at $1,21,36$, and 76 days of age of birds respectively (Supplementary table 1 and 3). The HI titers (Mean $\pm \mathrm{SD}$ ) of birds of group A (vaccinated), were 70.40 $\pm 035.054,89.60 \pm 35.054,153.60 \pm 57.243$, and $563.20 \pm$ 280.433 at 1, 20, 36 and 76 days of age respectively. For easy comparison this $\mathrm{HI}$ antibody titers (Mean $\pm \mathrm{SD}$ ) were converted into $\log _{2} \mathrm{HI}$ titers (Mean $\pm \mathrm{SD}$ ). The $\log _{2}$ value were $6.00 \pm 0.7071,6.40 \pm 0.5477,7.20 \pm 0.4472$, and $9.00 \pm$ 0.7071 , at $1,21,36$, and 76 days of age of birds respectively (Supplementary table 2 and 3 ).

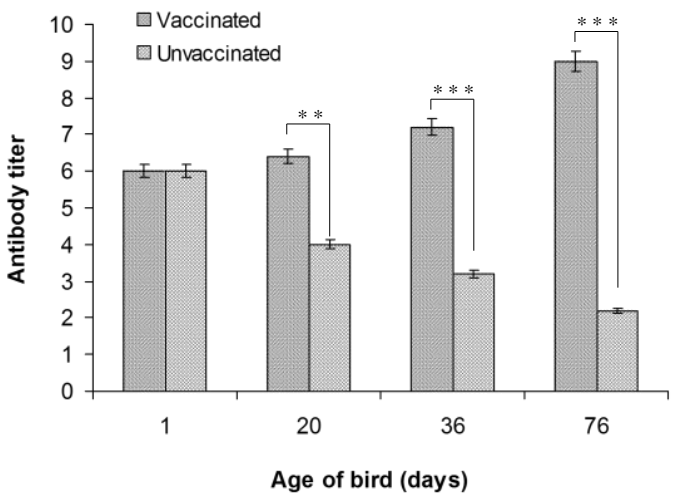

Fig 1: HI antibody titer ( $\log 2$ base) of vaccinated and non vaccinated birds. Chickens were immunized twice with BCRDV through i/o at 2 and 21 days of age and with RDV at 60 days of age through i/m route. Blood was drawn at $01,20,36$, and 76 days age of birds. HI antibody titers of the serum against NDV antigen were determined by HI test. ${ }^{* *} P=<0.01$ and ${ }^{* * * *} P=<0.001$

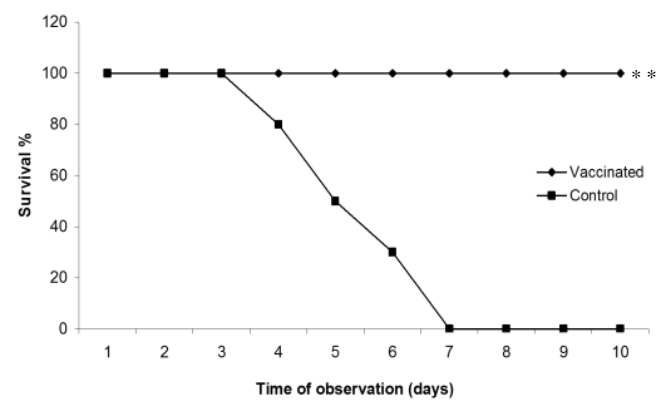

Fig 2: Survival rate of chicken following challenge infection (protection test) intranasally with virulent field isolate of NDV. Chickens were immunized twice with BCRDV through (i/o) route at 2 and 21 days of age and with RDV at 60 days of age through (i/m) route. Survival rate of chicken was monitored following intranasal challenge with $2 \mathrm{ELD}_{50}$ dose of NDV and observed for subsequent 10 days, where ${ }^{* *} P=$ $<0.01$ by Mantal-Cox log rank test.

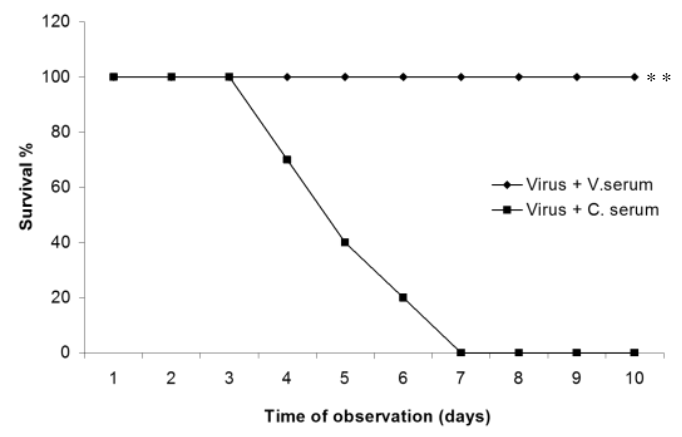

Fig 3. Survival rate of chicken following passive challenge test (passive protection test). Survival rate of chicken was monitored following intranasal inoculation of $2 \mathrm{ELD}_{50}$ dose of NDV with $200 \mu \mathrm{l}$ pooled immune serum or $200 \mu \mathrm{l}$ PBS and observed for subsequent 10 days, where ${ }^{* *} P=<0.01$ by Mantal-Cox log rank test.

\section{Protection test}

After the challenge, the mortality of challenged birds was monitored for subsequent 10 days. In case of vaccinated group, no signs of illness were observed and survived post-challenge infection throughout the period of observation (10 days). On the other hand, all the control birds 
challenged at the same day showed typical clinical sign of ND such as sneezing, gasping, greenish-watery diarrhea, drowsiness, and all birds succumbed within 10 days after challenge and also produced typical postmortem lesion. It appeared that birds vaccinated with BCRDV and RDV conferred $100 \%$ protection following virulent challenge (Fig. 2).

\section{Passive protection test}

After performing the passive protection test the mortality of all the tested birds was monitored for subsequent 10 days (Fig. 3). Virus mixed with nonimmunized serum administered birds were showed typical clinical signs of ND such as sneezing, gasping, greenish-watery diarrhea, drowsiness, and finally die within 10 days. On the other hand virus mixed with immunized serum administered birds were not expressed any sign of illness. That means no clinical sign of ND were showed within 10 days of observation period. This indicates $0.2 \mathrm{ml}$ of hyperimmune serum was able to neutralized $0.1 \mathrm{ml}$ of infective dose of virus as a result no ND was occurred.

\section{Discussion}

The experiment stated that the antibody titers of unvaccinated control group of birds gradually decreased from day 1 to day 76 of age (Fig. 1; Supplementary table 1,2 and 3). At one day age of chicks, before any vaccination the $\log 2 \mathrm{HI}$ antibody titers of MDA were $6.0 \pm 0.70$ which gradually declined to $2.20 \pm 0.44$ in day 76 . On day 20 , and 36 antibody titer were $4.00 \pm 0.7071$ and $3.20 \pm$ 0.44 respectively. On the other hand, in vaccinated group (group A), following primary vaccination with BCRDV (day 2), the antibody titers slightly increased at day 20 compared with maternal antibody which were recorded $6.0 \pm 0.70$ in $\log 2$ HI titers. Similarly, after secondary vaccination with BCRDV (day 21) antibody titer of serum that was obtained at day 36 was also slightly increased compared with day 20 and was recorded 6.20 \pm 0.4472 . From this result, it was clear that anti-body titer did not sharply increased after primary and secondary vaccination with BCRDV compared with MDA due to presence of high maternal antibody. The chicks used in this study had comparatively high maternal anti-body because the chicks were hatched from eggs of Hisex hens of reputed Phenix Hatchery Ltd., which follow intensive vaccination program against ND. This resulted in high maternal antibody titer; consequently antibody titers did not become sharply high after vaccination.

Third vaccination with RDV at 60 days of age through $\mathrm{i} / \mathrm{m}$, following primary and secondary vaccination with BCRDV via i/o exhibited sharply revealed high antibody titer of $9.00 \pm 0.7071$ (Fig. 1; Supplementary table 1, 2 and 3). Chowdhury et al. (1981) mentioned administration of live lentogenic vaccine (BCRDV) of F-strain by i/o inoculation at first week and 21 days old chicks followed by a live mesogenic vaccine (RDV) of $\mathrm{M}$ strain by $\mathrm{i} / \mathrm{m}$ route at day 60 and repeated at every 6 months interval provided better antibody titer. However, Kafi (2003) reported that vaccination schedule consisting of a live lentogenic vaccine followed by an inactivated or killed vaccine conferred higher and durable level of immunity. For this reason, the poultry breeders usually used a live lentogenic vaccine for the chick followed by an inactivated vaccine to obtain better immune response.
From this study it was also evidenced that $100 \%$ of the birds vaccinated with BCRDV and RDV were protected against challenge infection ( after 16 days post vaccination with RDV) with a virulent field isolate of NDV and the unvaccinated control group of birds had no protection capacity (Fig. 2). The challenge test demonstrated that the BCRDV and RDV conferred higher protection to birds against field virus. Rahman et al. (2004) reported that secondary vaccination with BCRDV provided $100 \%$ protection at 38 days of age. Analogously, Chowdhury et al. (1981) found that day-old to 12 days old chicks administered with $B_{1}$ vaccine by $i / o$ or intranasal $(i / n)$ route revealed $80 \%$ protection with virulent NDV at 3 weeks after vaccination. Chauhan and Roy (1996) showed that chickens vaccinated with the lyophilized Newcastle disease (ND) $F$ vaccine at 1,21 and 42 days were protected when challenged at $14,35,56$ and 70 days with the virulent strain of Newcastle disease virus (NDV) administered intramuscularly, intranasally, or by contact. Produced immune response was also confirmed by the passive protection test in unvaccinated control birds. The results of the passive protection test indicated that produced humoral antibody was sufficient for neutralization of infective dose of virulent virus consequently disease did not occur (Fig. 3). Finally, present study indicated that administration of the BCRDV followed by RDV stimulated satisfactory antibody response. Though primary and secondary vaccination with BCRDV produced low level of antibody, but booster vaccination with RDV induced sufficient antibody titer to protect virulent challenge.

\section{Conclusion}

This study revealed that administration of BCRDV and RDV produced by LRI was effective for protection against virulent challenge with a field isolate of NDV. It was observed that following challenge infection with a virulent field isolate of virus, $100 \%$ of the birds vaccinated with BCRDV and RDV were refractory to virulent challenge while the unvaccinated control group of birds had no such protective potentiality. In addition, sera from vaccinated birds were able to neutralize the infective dose of virulent virus that was assessed by passive protection test. Both BCRDV and RDV are effective for protection against natural field virus but in presence of high maternal antibody titers chicks should be vaccinated with BCRDV after two weeks of age. Similarly, secondary or booster vaccination with BCRDV should be rescheduled from the currently practiced 21 days of age.

\section{Acknowledgements}

We thank the Department of Microbiology and Hygiene where the research work was performed. We humbly desire to convey our deepest gratitude to National Agricultural Technology Project (NATP) as well as Department of Livestock Services, Ministry of Fisheries and Livestock, Government of the People's Republic of Bangladesh, for supporting the research work and financial grant.

\section{References}

Anon, 1971. Methods for examining poultry biologics and for identification and quantifying avian pathogens. Newcastle disease, US National Academy of sciences, Wasinghton DC, pp: 66. 
Beard CN and RP Hanson, 1984. Newcastle disease. In Diseases of Poultry. $8^{\text {th }}$ Ed. Edited by Hofstad, M.S. Iowa State University Press, Ames, Iwoa. USA. pp: 452-470.

Brown C, DJ King and B Seal, 1999. Pathogenesis of Newcastle disease in chickens experimentally infected with viruses of different virulence. Vet Pathol, 36:125132.

Chauhan HVS and S Roy, 1996. Poultry Diseases, Diagnosis and Treatment. $2^{\text {nd }}$ Ed. New Age International (P) Limited Publishers, New Delhi, pp: 58-64.

Cheville NF, H Stone, J Riley and AE Ritchie, 1972. Pathogenesis of virulent Newcastle disease in chickens. J Am Vet Med Assoc, 161: 169-179.

Chowdhury TIMF, AJ Sarker, MM Amin and WIMA Hossain, 1981. Studies of Newcastle disease in Bangladesh. Bangl Vet J, 15: 1-9.

Chowdhury SI, TIMFR Chowdhury, AJ Sarker, MM Amin and WIMA Hossain, 1982. Studies on Newcastle disease in Bangladesh. A research report. 2. The ro- le of residual maternal antibody on immune response and selection of an optimum age for primary vaccination of chicks. pp: 12-22.

Cottral GE, 1978. Manual of standardized methods for veterinary microbiology. Cornell University press. Ithaca, NY. pp: 47-52.

Kafi A, 2003. Vaccination studies on use of Ranikhet inactivated vaccine combined with $\mathrm{V}_{4} \mathrm{HR}$ or BCRDV MS Thesis, Department of Microbiology and Hygiene, BAU, Mymensingh-2202.

Rahman MB, MM Rahman, M Rahman, SML Kabir, KHMNH Nazir and MM Amin, 2004. Efficacy of V4HR Newcastle disease (V4HR-ND) vaccine in broiler birds in Bangladesh. Int J Poult Sci, 3: 365-368.

Reed LJ and LH Muench, 1938. A simple method of estimating fifty percent end points. Am J Hygi, 27: 493-497.

Zhuo Z, M Chen, ZQ Zhou and MX Chen, 1998. Discussion on the causes for the outbreaks of IBD in immunized chicken flocks. Chi J Vet Med, 24:14. 